\title{
Effect of tendon hydrogel on healing of tendon injury
}

\author{
ZHENG CHEN, YONGJUN RUI, YAJUN XU, QUANRONG ZHANG, \\ ZHENZHONG SUN, JIANDONG ZHOU and XUEMING CHEN
}

\begin{abstract}
Department of Bone Surgery, Ninth People's Hospital of Wuxi City, Wuxi, Jiangsu 214062, P.R. China
\end{abstract}
Received September 11, 2015; Accepted November 4, 2016

DOI: $10.3892 /$ etm.2017.5020

\begin{abstract}
Hand trauma arising from postoperative acute or chronic tendon injuries leads to delayed union and is one of the greatest challenges in clinical practice. The present study hypothesized that an extracellular matrix hydrogel made from tendons can promote tendon healing and improve tissue regeneration. To verify this, $36 \mathrm{Wistar}$ rats were subjected to bilateral full-thickness injury of their Achilles tendons, starting from the heel bone along the center line to remove a segment of $5 \mathrm{~mm}$ in length and $0.5 \mathrm{~mm}$ in width. On the sites of injury, hydrogel was injected on one side, while the contralateral side was injected with an equal volume of normal saline. At 2, 4 and 8 weeks after the operation, bilateral tendons were subjected to biomechanical tests to determine the ultimate failure load, tensile strength and toughness, and cross-sectional slices of the tendons were subjected to histological analysis. The results indicated that after 2 weeks, the hydrogel and control group showed no significant difference in terms of ultimate load $(\mathrm{P}=0.15)$ ultimate tensile stress $(\mathrm{P}=0.42)$ and toughness $(\mathrm{P}=0.76)$. At 4 weeks following surgery, the failure load in the hydrogel group was significantly higher than that in the control group $(74.8 \pm 14.2$ vs. $58.4 \pm 11.6$, $\mathrm{P}=0.02)$, while there were no significant differences in the ultimate tensile stress $(\mathrm{P}=0.63)$ and toughness $(\mathrm{P}=0.08)$. At 8 postoperative weeks, the abovementioned parameters showed no significant difference between the groups $(\mathrm{P}=0.15$, 0.39 and 0.75 , respectively). In conclusion, the tendon-derived extracellular matrix hydrogel was able to significantly improve tendon strength at 4 weeks after injury in terms of increasing the ultimate failure load. Hydrogel applied immediately after tendon injury can enhance the type-I collagen content. The present study therefore provided a basis for further exploration of the application of extracellular matrix hydrogel to promote tendon healing in the clinic.
\end{abstract}

Correspondence to: Dr Yongjun Rui, Department of Bone Surgery, Ninth People's Hospital of Wuxi City, 999 Liangqi Road, Wuxi, Jiangsu 214062, P.R. China

E-mail: ruiyongjwx@yeah.net

Key words: Hydrogel, hand trauma, tendon injury, healing

\section{Introduction}

Tendon repair is an important step in hand surgery and other types of plastic surgery for a long time period post-surgery, tendons cannot be subjected to strain and their biomechanical properties are aberrant; micro tears, which may ultimately lead to full-thickness injury of tendon, can be characterized by microscopy $(1,2)$. Common tendon injuries include the Achilles tendon, rotator cuff tendon and elbow lateral condyle, and due to their similar features and poor prognosis, numerous studies have assessed strategies to promote tendon healing, mainly including platelet-rich plasma, whole-blood injections, growth hormones and stem cells (3). Tendon healing after injury is usually affected by inflammation, collagen production and reshaping of. In the early stages after tendon damage, mononuclear cells and macrophages are activated, which release biomolecular factors promoting tendon cell proliferation and increase in collagen synthesis; these processes occurring as early as possible may provide sufficient mechanical strength to enable patients to perform basic activities. Studies have shown that early activity is of great significance for tendon performance and associated prognosis; early healing after tendon injury is therefore key (4-6). Tendon-derived extracellular matrix hydrogels containing connective tissue-specific proteins, including collagen I, can deliver tendon cells to the damage location and provide an environment supporting cell proliferation $(7,8)$. Previous studies on rats showed that subcutaneous hydrogel injection was well tolerated with no nodules or allergic reaction and gradual degradation after 4 weeks $(7,8)$. Based on these preliminary studies, the present study explored the ability of a tendon-derived hydrogel to promote tendon healing in rats after injury. The present study paved the road for the implementation of hydrogels for tendon healing in clinical practice.

\section{Materials and methods}

Tissue decellularization. The musculi flexor digitorum profundus, musculus flexor digitorum superficialis and flexor pollicis longus tendon were obtained from 36 rats (male, average weight, $350 \mathrm{~g}, 6-8$ weeks) cadavers frozen directly after sacrifice. This study was approved by the ethics committee of the Ninth People's Hospital of Wuxi City, Wuxi, China. According to a previously described method (8), the epitenon, vaginae synoviales and musculature were extracted. The 
superficial flexor tendon was cut near the bifurcation at $2 \mathrm{~cm}$ from the end. The musculus flexor digitorum superficialis and flexor pollicis longus tendon were cut off near the bone tendon junction at $1 \mathrm{~cm}$ from the end. The tendons obtained were subjected to acellular treatment and subsequently immersed in $0.1 \%$ EDTA solution for $4 \mathrm{~h}(8)$. The tendons were then incubated in $0.1 \%$ lauryl sodium sulfate and $0.1 \%$ EDTA solution at room temperature for $24 \mathrm{~h}$ with stirring, followed by washing with phosphate buffer. The tendons were then frozen at $-80^{\circ} \mathrm{C}$. The acellular tendon tissue was then freeze-dried, ground into powder and kept at $4^{\circ} \mathrm{C}$ until use.

Preparation of tendon matrix hydrogel. The tendon powder was incubated with $1 \mathrm{mg} / \mathrm{ml}$ pepsin (Sigma-Aldrich, Merck $\mathrm{KGaG}$, Darmstadt, Germany), 0.02M hydrochloric acid and aquae sterilisata ( $\mathrm{pH} 2.2$; powder concentration, $20 \mathrm{mg} / \mathrm{ml}$ ) to digest the extracellular matrix. Following incubation at room temperature for $24 \mathrm{~h}$ with constant stirring, the sample was placed on ice and sodium carbonate hydrate was added to increase the $\mathrm{pH}$ to 8.0 to inactivate pepsin. Finally, 10 volumes of phosphate-buffered saline (PBS, adjusting $\mathrm{pH}$ 7.4) were added to obtain an isoosmotic solution.

Operative procedure and tendon treatment. A total of 36 Wistar rats (male, average weight, $350 \mathrm{~g}$, 6-8 weeks) were obtained from the animal center of Ninth People's Hospital, and were fed at room temperature and 40-50\% humidity, and with 12-h light/dark, and ad libitum access to food and water. The rats were fixed in the supine position after being anaesthetized by $2 \%$ isoflurane (Sigma-Aldrich; Merck $\mathrm{KGaG})$. This study was approved by the Ethics Committee of the Ninth People's Hospital of Wuxi City (Wuxi, China). Following anti-bacterial treatment with buprenorphine and enrofloxacin (Sigma-Aldrich; Merck KGaG), a 1-cm curve cut was made around the Achilles tendon to ensure the completeness of the tendon. The tendon was carefully separated to avoid damaging the neryus cutaneus surae medialis and major vessels. Cyanoacrylate was used to bond two \#15 surgical knives together to keep the incision parallel. Under a microscope, a damaged area of $0.5 \mathrm{~mm}$ in width and $5 \mathrm{~mm}$ in length was generated, reaching from the calcaneus to the central tendon of the bilateral lower extremities of each rat (Figs. 1 and 2). The site of injury on the left side was injected with $0.05 \mathrm{ml}$ tendon hydrogel, while the right-hand side was injected with $0.05 \mathrm{ml}$ phosphate-buffered saline as the control. The wound was then sutured with \#4 absorb string (Ethicon, Inc., Somerville, NJ, USA). According to the findings of a preliminary experiment (8), animals were anaesthetized using $1 \mathrm{ml} 2 \%$ isoflurane (20 mg/rat; Sigma-Aldrich; Merck KGaG) and then were sacrificed by decapitation at 2, 4 and 8 weeks after the surgery to obtain the tendons. A total of 10 tendon specimens were used for biomechanical performance tests at each time-point and 2 specimens were used for histopathological analysis.

Biomechanical performance test. The mini Bionix tset system (MTS Systems Corp., Eden Prairie, MN, USA) was used as a test load cell. From the rat feet obtained, the muscle was removed to expose the tendon junction. The foot was fixed, and abrasive paper and cyanoacrylate were used to fix the

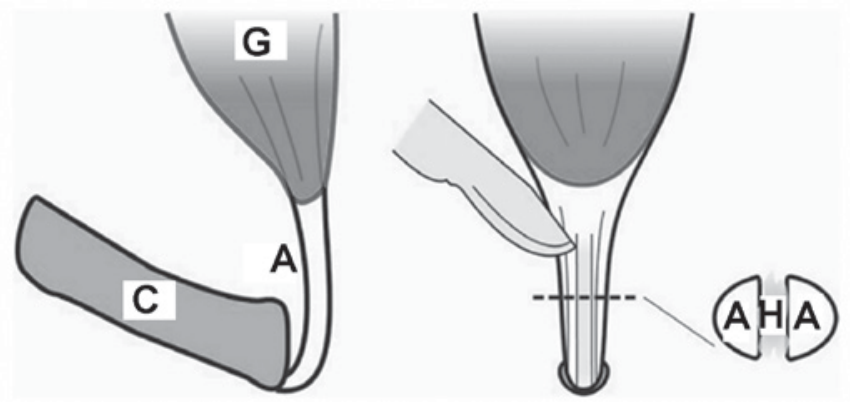

Figure 1. Illustration of the location and mode of surgery. C, calcaneus; G, gastrocnemius; A, Achilles tendon; H, hydrogel.

muscle-tendon junction (Fig. 3). Orthogonal images were captured with a digital camera (8.2 megapixels; Canon EOS 30D; Canon, Tokyo, Japan) and a digital caliper was used to determine the size of the tendon. Image $\mathrm{J}$ software version 2.0 (National Institutes of Health, Bethesda, MD, USA) was used to calculate the cross-sectional area and ultimate tensile stress of each tendon. At a tensile speed of $0.5 \mathrm{~mm} / \mathrm{sec}$, the load was increased until the destruction of the tendon and the ultimate failure load was recorded. The ultimate tensile stress yas determined by limiting load and cross-sectional area (8). Tendon toughness was evaluated via a force/displacement curve.

Histopathological analysis. The obtained tendon tissue was fixed for 10 min in $10 \%$ formalin (Sigma-Aldrich; Merck $\mathrm{KGaG})$ atroom temperature and embedded in ceresin wax and then cut into slices $(6 \mu \mathrm{m})$. The morphological characteristics of the tendons were evaluated by hematoxylin and eosin staining. Adjacent sections were stained with Sirius red solution (Sigma-Aldrich; Merck $\mathrm{KGaG}$ ) to determine the content of type-I and type-III collagen protein. Images were captured under a fluorescence microscope (Model: D-35578; Leica Microsystems, Wetzlar, Germany).

Statistical analysis. All of the data were analyzed by using the SPSS software 18.0 (SPSS Inc., Chicago, IL, USA). Comparisons among groups were performed by the paired Student's t-test. Values are expressed as the mean \pm standard deviation. $\mathrm{P}<0.05$ was considered to indicate a statistically significant difference.

\section{Results}

Biomechanical test. For every biomechanical test, one rat tendon was rolled out from the forceps holder. The connection of the tendon muscle was broken, so that it was excluded. The results showed that 2 weeks after the operation, almost all tendon rupture positions were located in the tendon itself. By contrast at 4 and 8 weeks post-surgery, ruptures of the muscle-tendon junction were common (Table I). At 2 weeks after the operation, no obvious difference in ultimate failure load, ultimate tensile stress and toughness was observed between the two groups $(\mathrm{P}=0.151,0.424$ and 0.760 , respectively). At 4 weeds after the operation, the ultimate failure load in the experimental group was increased by $28 \%$ of that of the control group (74.8 \pm 11.6 vs. $58.4 \pm 14.2 \mathrm{~N}$; $\mathrm{P}=0.016$; Fig. 4), 
Table I. Results of biomechanical tests.

\begin{tabular}{|c|c|c|c|c|c|c|}
\hline Group & Treatment & $\begin{array}{l}\text { Mechanical tests/ } \\
\text { tissue analyses (n) }\end{array}$ & $\begin{array}{c}\text { Ultimate } \\
\text { failure load }(\mathrm{N})\end{array}$ & $\begin{array}{l}\text { Ultimate tensile } \\
\text { stress }\left(\mathrm{N} / \mathrm{mm}^{2}\right)\end{array}$ & $\begin{array}{l}\text { Toughness } \\
(\mathrm{N} / \mathrm{mm})\end{array}$ & $\begin{array}{c}\text { Tendon damage } \\
\text { location }\end{array}$ \\
\hline \multirow[t]{2}{*}{1} & $2 \mathrm{w}$, hydrogel & $4 / 1$ & $45.2 \pm 8.6$ & $1.6 \pm 0.6$ & $11.4 \pm 3.7$ & 1TBI/8T/0B \\
\hline & $2 \mathrm{w}$, no hydrogel & $9 / 2$ & $39.8 \pm 6.3$ & $1.4 \pm 0.2$ & $10.9 \pm 2.7$ & 2TBI/7T/0B \\
\hline \multirow[t]{2}{*}{2} & $4 \mathrm{w}$, hydrogel & $9 / 2$ & $74.8 \pm 11.6^{\mathrm{a}}$ & $3.0 \pm 0.7$ & $17.9 \pm 3.5$ & $5 \mathrm{TBI} / 4 \mathrm{~T} / 0 \mathrm{~B}$ \\
\hline & $4 \mathrm{w}$, no hydrogel & $9 / 2$ & $58.4 \pm 14.2^{\mathrm{a}}$ & $2.8 \pm 1.0$ & $14.7 \pm 3.9$ & 7TBI/2T/0B \\
\hline \multirow[t]{2}{*}{3} & $8 \mathrm{w}$, hydrogel & $9 / 2$ & $83.5 \pm 13.7$ & $2.9 \pm 0.7$ & $17.7 \pm 2.7$ & $5 \mathrm{TBI} / 4 \mathrm{~T} / 0 \mathrm{~B}$ \\
\hline & $8 \mathrm{w}$, no hydrogel & $9 / 2$ & $74.0 \pm 13.2$ & $2.6 \pm 0.7$ & $17.3 \pm 4.5$ & $6 \mathrm{TBI} / 3 \mathrm{~T} / 0 \mathrm{~B}$ \\
\hline
\end{tabular}

${ }^{\text {ap }}<0.05$ represents the values compared to the $2 \mathrm{w}$, hydrogel or $2 \mathrm{w}$, no hydrogel. TBI, the junction of tendon and bone; $\mathrm{T}$, tendon; $\mathrm{B}, \mathrm{bone} ; \mathrm{w}$, weeks.

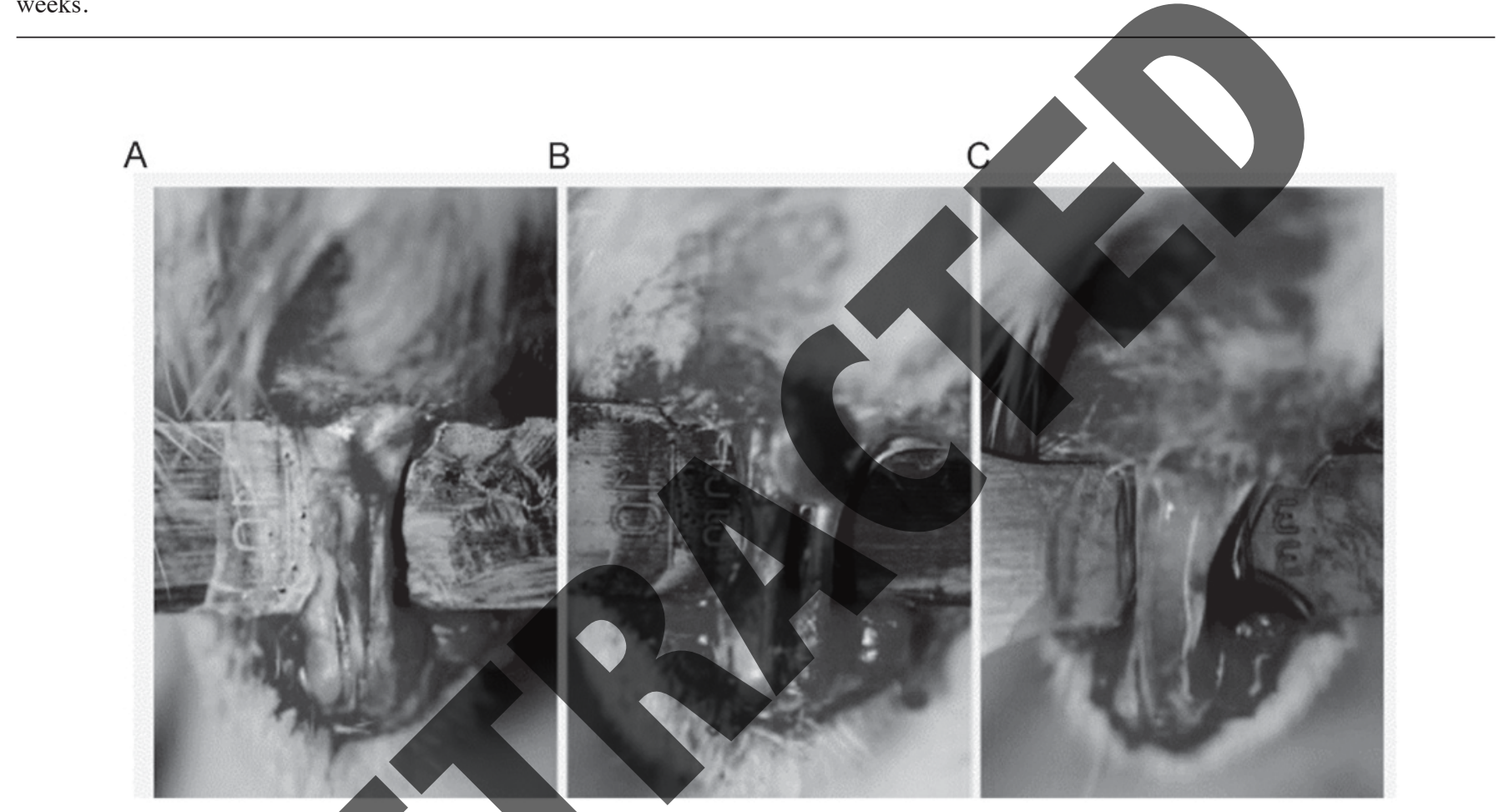

Figure 2. Operation procedure. (A) The tendon was cut on both sides in a parallel fashion. (B) The damaged area except for the central part was put into shape under the microscope and (C) filled with hydrogel. Magnification, x200.

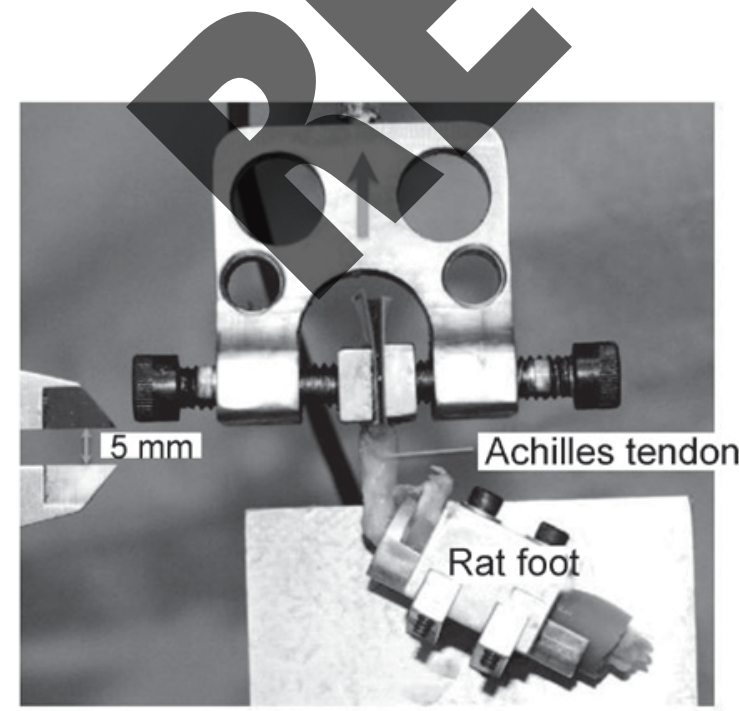

Figure 3. Biomechanical test. For measurement of the ultimate failure load, tensile strength and toughness, a load was applied at the distance shown by the vernier caliper $(5 \mathrm{~mm})$. The arrowhead shows the direction of the applied load.

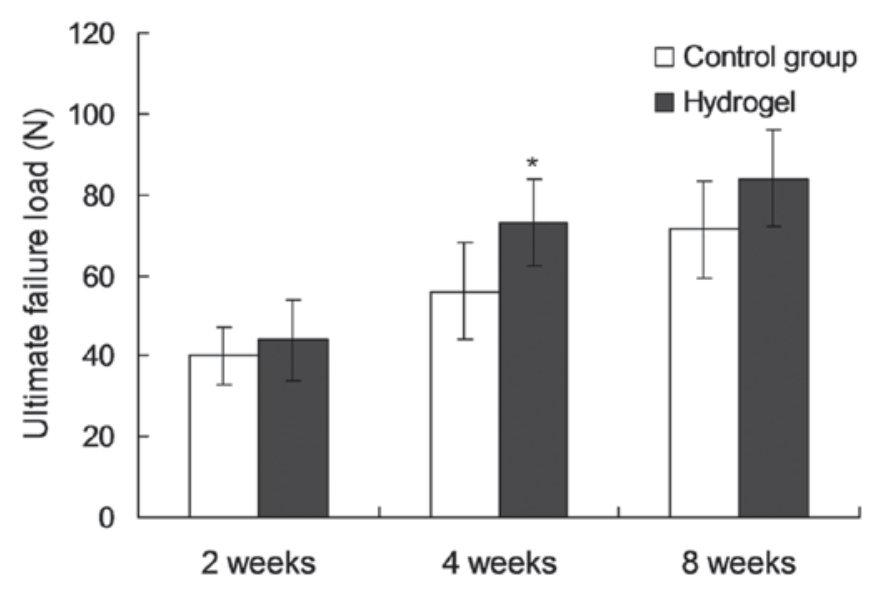

Figure 4. Ultimate failure load for each time point. ${ }^{*} \mathrm{P}<0.05$ vs. Control group. 
A

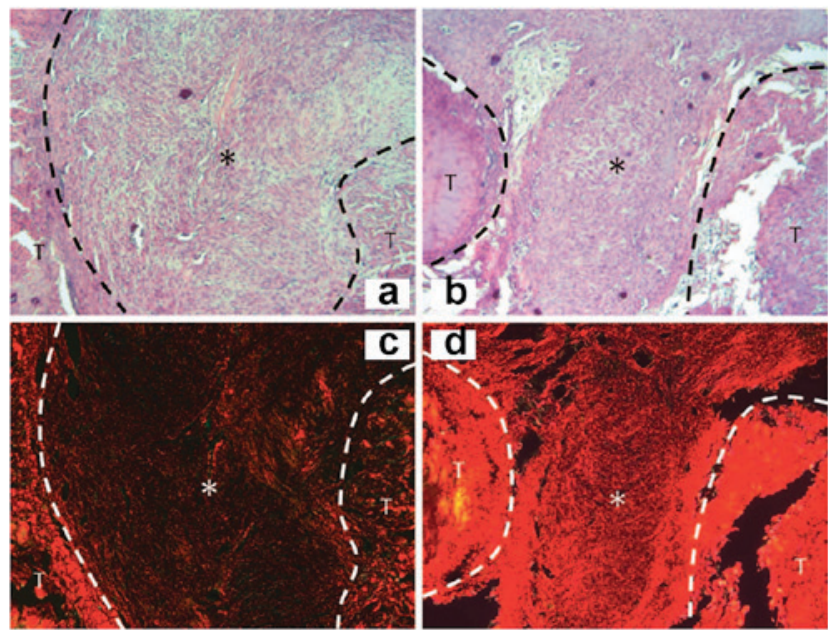

B

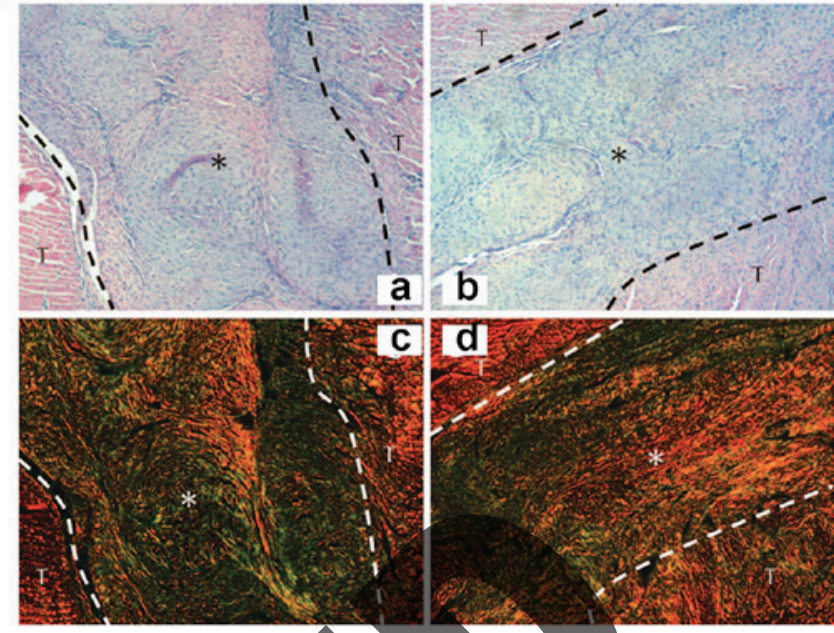

Figure 5. H\&E and Sirius red staining at 2 and 4 weeks after the operation. (A) H\&E staining of (a) the control and (b) the experimental group as well as Sirius red staining of (c) the control and (d) the experimental group at 2 weeks after operation. Type I collagen protein (red staining) in the experimental group was shown to be higher than that in the control group. (B) H\&E staining of (a) the control and (b) the experimental group as well as Sirus red staining of (c) the control and (d) the experimental group at 4 weeks after operation. Type III collagen protein (green and yellow) in the control group was obvious higher than that in the experimental group. Furthermore, type I collagen protein (red) in the experimental group was higher than that in the control group. T, tendon; ", injury position; H\&E, hematoxylin and eosin. Magnification, x200.
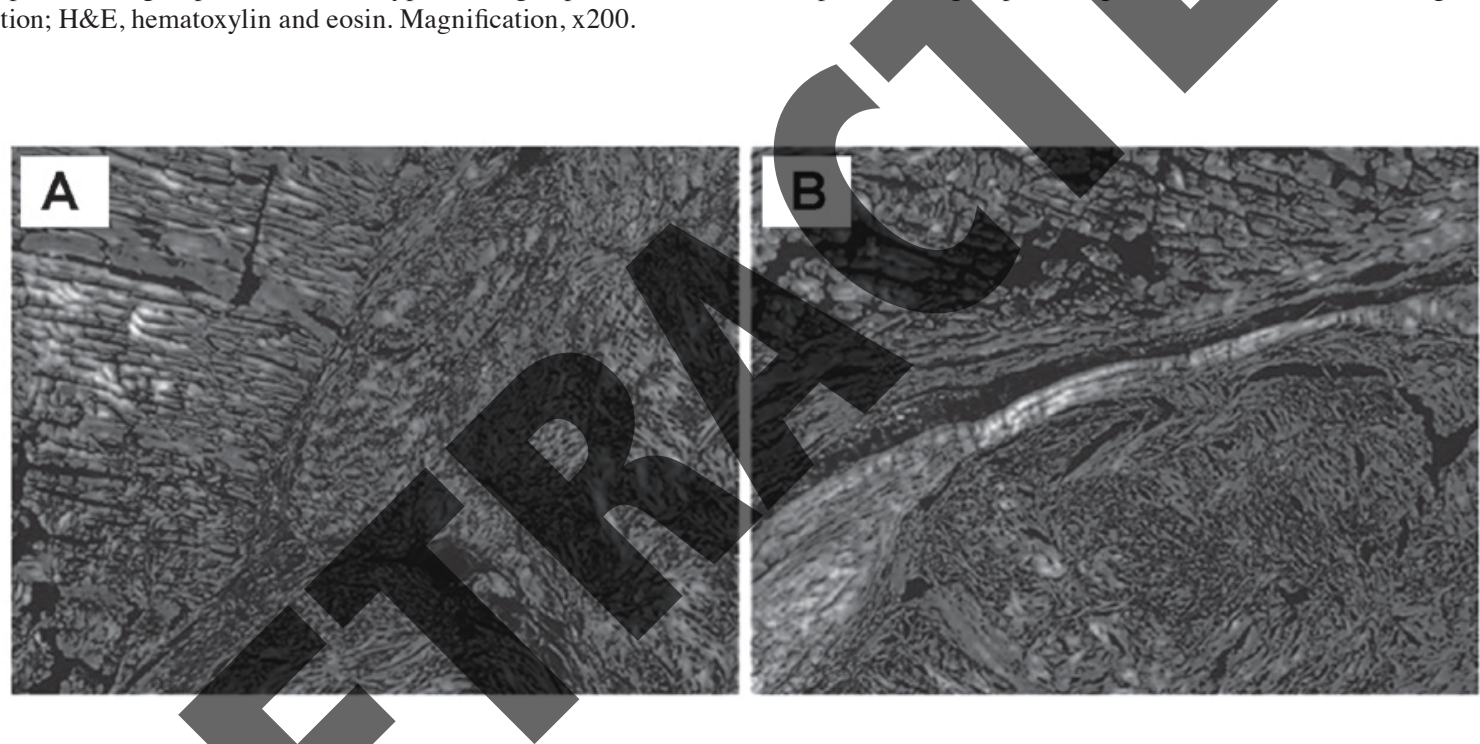

Figure 6. At 8 weeks after the operation, the contentrof type I collagen protein in (A) control group and (B) hydrogel group was almost the same. The damaged area has become invisible. The samples were identical, while an incision/scar is still visible in the experimental group. However, only one animal was assessed in here. Magnification.

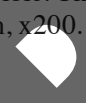

while the ultimate tensile stress and toughness were not significantly different between the two groups $(\mathrm{P}=0.634$ and 0.082 , respectively). At 8 weeks post-surgery, the ultimate failure load, ultimate tensile stress and toughness did not show any significantly differences between the two groups $(\mathrm{P}=0.156$, 0.385 and 0.748 , respectively).

Histopathological analysis. At 2 and 4 weeks after the operation, the cross-section of the tendons of the two groups was almost identical. In the hydrogel-treated group, the collagen aggregation in the injury position was visible (Figs. 5 and 6). At 2 weeks post-surgery, Sirius red staining showed that the type I collagen content in the experimental group was obviously higher than that in the control group (Fig. 5A). At week 4, the difference in collagen content in hydrogel group was more significant compared to control group,

which suggests that hydrogel applied immediately after tendon injury can enhance the type-I collagen fibers $(\mathrm{P}<0.05$, Fig. 5B). Meanwhile, type III collagen protein (green and yellow) in the control group was obvious higher than that in the experimental group $(\mathrm{P}<0.05$, Fig. 5). Furthermore, type I collagen protein (red) in the experimental group was higher than that in the control $(\mathrm{P}<0.05$, Fig. 5). At 8 weeks, no obvious difference was found in the collagen content between the two groups (Fig. 6).

\section{Discussion}

The results of the present study confirmed the former hypothesis that a tendon-derived extracellular matrix hydrogel can improve tissue regeneration and promote tendon healing. The use of tissue-derived hydrogel has been previously 
reported. Ventricle-derived extracellular matrix hydrogel has been applied in a model of cardiac damage, which improved the repair of myocardial cells and local metabolism, and skeletal muscle-derived extracellular matrix hydrogel has been applied in a model hindlimb ischemia which enhanced the distribution of skeletal muscle cells along the neovascularization $(8,9)$. However, collagen hydrogel synthesized in vitro did not obviously improve the healing of tendon injury (10). In the present study, a hydrogel not only containing the matrix component of tendons, but also its proteins and biological factors, was prepared and applied. To the best of our knowledge, the present study was the first to prepare tendon matrix hydrogels and apply them to an animal model of tendon injury.

The biocompatibility of the extracellular matrix hydrogel has been shown to be good with degradation occurring after 4 weeks. The results of the present study showed that extracellular matrix hydrogel provides a suitable environment for cell metabolism and healing. For instance, strengthening of the phagocytic function of macrophages is beneficial for tissue remodeling (9). In the first stage of tendon healing, macrophages destroy and take up necrotic tissue, and release extracellular matrix, oligosaccharide derivatives and other factors. Macrophages can be used as a vasoactive and chemotactic factor to promote the aggregation of stem and progenitor cells. Furthermore, collagen synthesis is increased in the early stages of injury to repair damaged tissue (11). Certain studies have indicated that weakening of the tendon strength during the healing process is due to the natural collagen protein I being replaced by the thinner type III collagenous fibers $(12,13)$. Sirius red staining at 4 weeks post-surgery/showed that in the hydrogel group, the ratio of type I vs. type III collagen was obviously higher than that in the control group, which explains for the higher ultimate failure load in the hydroge group. The type I collagen content was therefore consistent with the mechanical strength of the tendon. 4 weeks later, the hydrogel-treated tendon was thicker and more solid in hydrogel group (one animal) and the intersecting surface was greater compared to the control group. The toughness of the tendon arises from cross-linked collagenous fibers in the final stage of healing (14), Therefore, in the present study, not all of the differences were significant at different time-point (such as 4 and 8 weeks). The effect of the hydrogel was mainly shown in the early stages of injury and the hydrogel, as it was degraded after 4 weeks. Therefore, its effect of promoting tissue repair gradually decreased with time. The operation wound in the two groups was still visible at 8 weeks and the scars were covered by type I collagen, however, only one animal was assessed. The results indicated that the process of tissue repair and remodeling was almost finished at 8 weeks after tendon injury.

In clinical practice, the main application advantages of hydrogel may be that it accelerates the healing process of tendon injury in the early stage, enabling patients to perform early functional exercise. Numerous studies have shown that application of an appropriate mechanical force can promote fibroblast proliferation and the synthesis and arrangement of the original collagen protein $(15,16)$. The hydrogel can be gathered at the physiological temperature, so that the targeted application to part of the tendon injury can be used by minimally invasive injection to improve the prognosis. Furthermore, substances including growth factors, platelet-rich plasma and stem cells can be mixed with hydrogel for injection; the efficacy of such preparations requires evaluation by further studies.

Of note, the present study had certain limitations. Sirius red staining used for the quantitative assessment of collagen content only provided one possible explanation for the differences in tendon strength demonstrated by the biomechanical test. However, the findings only provided limited information, as only one rat per group was used for histopathological analysis at each time-point. In further studies, analysis of changes in structure, collagen synthesis and content of associated biological factors will be helpful to confirm the effect of hydrogel on tendon healing. In the present study, a rat model of tendon injury was used, while there may be differences among various injury sites and species. Furthermore, an animal experiment using a larger sample is required for further comparison and confirmation of the results.

In conclusion, the present study showed that a tendon-derived extracellular matrix hydrogel was able to significantly improve the ultimate failure load of injured tendons from rats at 4 weeks after surgery. It was therefore suggested that the hydrogel may promote tendon healing. The present study paved the road for the development of a novel preparation for the clinical treatment of tendon injuries.

\section{References}

Yu YD, Zhang YZ, Bi WD and Wu T: Functional sensory function recovery of random-pattern abdominal skin flap in the repair of flingertip skin defects. Exp Ther Med 5: 830-834, 2013.

2. Neviaser A, Andarawis-Puri N and Flatow E: Basic mechanisms of tendon fatigue damage. J Shoulder Elbow Surg 21: 158-163, 2012.

3. Sharma P and Maffulli N: Biology of tendon injury: Healing, modeling and remodeling. J Musculoskelet Neuronal Interact 6: 181-190, 2006.

4. Longo UG, Lamberti A, Maffulli N and Denaro V: Tissue engineered biological augmentation for tendon healing: A systematic review. Br Med Bull 98: 31-59, 2011

5. Beck J, Evans D, Tonino PM, Yong S and Callaci JJ: The biomechanical and histologic effects of platelet-rich plasma on rat rotator cuff repairs. Am J Sports Med 40: 2037-2044, 2012.

6. Virchenko $\mathrm{O}$ and Aspenberg P: How can one platelet injection after tendon injury lead to a stronger tendon after 4 weeks? Interplay between early regeneration and mechanical stimulation. Acta Orthop 77: 806-812, 2006.

7. Sato D, Takahara M, Narita A, Yamakawa J, Hashimoto J, Ishikawa $\mathrm{H}$ and Ogino $\mathrm{T}$ : Effect of platelet-rich plasma with fibrin matrix on healing of intrasynovial flexor tendons. J Hand Surg Am 37: 1356-1363, 2012.

8. Kampa RJ and Connell DA: Treatment of tendinopathy: Is there a role for autologous whole blood and platelet rich plasma injection? Int J Clin Pract 64: 1813-1823, 2010.

9. Andersson T, Eliasson P and Aspenberg P: Growth hormone does not stimulate early healing in rat tendons. Int J Sports Med 33: 240-243, 2012.

10. Ngo M, Pham H, Longaker MT and Chang J: Differential expression of transforming growth factor-beta receptors in a rabbit zone II flexor tendon wound healing model. Plast Reconstr Surg 108: 1260-1267, 2001.

11. Chong AK, Ang AD, Goh JC, Hui JH, Lim AY, Lee EH and Lim BH: Bone marrow-derived mesenchymal stem cells influence early tendon-healing in a rabbit achilles tendon model. J Bone Joint Surg Am 89: 74-81, 2007. 
12. Okamoto N, Kushida T, Oe K, Umeda M, Ikehara S and Iida H: Treating Achilles tendon rupture in rats with bone-marrow-cell transplantation therapy. J Bone Joint Surg Am 92: 2776-2784, 2010.

13. Sharma $\mathrm{P}$ and Maffulli N: Tendon injury and tendinopathy: Healing and repair. J Bone Joint Surg Am 87: 187-202, 2005.

14. Huss FR, Nyman E, Bolin JS and Kratz G: Use of macroporous gelatine spheres as a biodegradable scaffold for guided tissue regeneration of healthy dermis in humans: An in vivo study. J Plast Reconstr Aesthet Surg 63: 848-857, 2010.
15. Wolf MT, Daly KA, Brennan-Pierce EP, Johnson SA, Carruthers CA, D'Amore A, Nagarkar SP, Velankar SS and Badylak SF: A hydrogel derived from decellularized dermal extracellular matrix. Biomaterials 33: 7028-7038, 2012.

16. Singelyn JM, Dequach JA, Seif-Naraghi SB, Littlefield RB, Schup-Magoffin PJ and Christman KL: Naturally derived myocardial matrix as an injectable scaffold for cardiac tissue engineering. Biomaterials 30: 5409-5416, 2009.

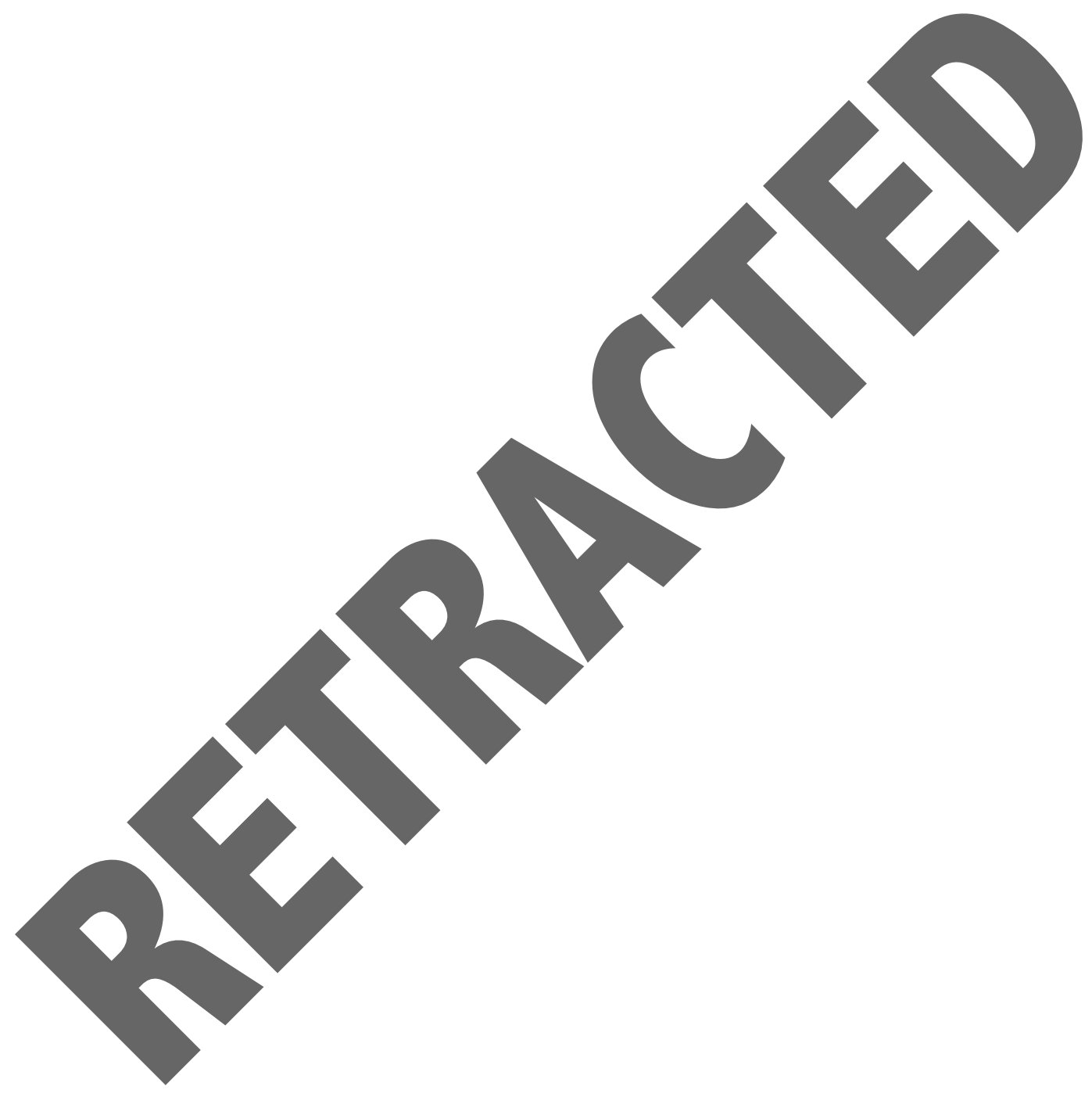

